\title{
CARRIER HEATING EFFICIENCY IN OPTICALLY DETECTED CYCLOTRON RESONANCE EXPERIMENT*
}

\author{
S. Dedulewicz ${ }^{\dagger}$ AND M. GodlewsKi \\ Institute of Physics, Polish Academy of Sciences \\ Al. Lotników 32/46, 02-668 Warszawa, Poland
}

\begin{abstract}
Monte Carlo calculations of carrier heating efficiency in optically detected cyclotron resonance experiment are presented. It is shown that electrons accelerated by microwa ve electric field gain energy sufficient for impact ionization of shallow centers and for exciton dissociation. It is also explained why very sharp thresholds for impact processes were observed in the optically detected cyclotron resonance investigations.

PACS numbers: 78.70.Gq, 72.10.Di, 72.10.Fk
\end{abstract}

\section{Introduction}

The impact ionization process, caused by the microwave field accelerated free carriers, can be responsible for the detection of the cyclotron resonance (CR) absorption via the photoluminescence (PL). The first optically detected cyclotron resonance (ODCR) studies confirmed in fact an important role of this mechanism [1-5]. An unexpected feature of these studies was observation of very sharp thresholds for the impact ionization processes [6].

This aspect of the ODCR experiment is analyzed in the present work. The carrier heating efficiency by the microwave field is calculated for material parameters of the $\mathrm{GaAs}$ and for the $4 \mathrm{~K}$ sample temperature. These calculations were performed for two extreme doping limits, i.e., for lightly and heavily doped sample. The performed Monte Carlo calculations indicate that microwave heated free electrons can accumulate energy sufficient for impact ionization of most of the shallow impurities or for dissociation of free and bound excitons in common semiconducting materials.

The distribution function of the mean electron energy was calculated. We explain the observed sharp thresholds for the impact processes and the resulting high spectral resolution of the ODCR study by the sharp distribution function of the mean electron energy occurring for electron heating by low magnitude and high frequency electric field component of the microwaves.

*This work was partly supported by the research grant no. 204769101 of the Committee for Scientific Research.

IOn leave from Semiconductor Physics Institute, A. Goštauto 11, Vilnius, Lithuania. 


\section{Monte Carlo calculations}

The Monte Carlo calculations performed are essentially of the type described by Fawcett et al. [7]. This calculations were performed under following approximations: phonon distribution in the crystal is not affected by electric field applied, bottom of the conduction band is parabolic, inter-valley scattering is not present. These assumptions are justified for common III-V and II-VI semiconductors, for which ODCR studies were performed. Simulations were performed for material parameters of GaAs, which are best known and are typical of other III-V compounds [8].

First, we determined the relative efficiencies of different possible carrier scattering mechanisms. For expected doping levels and for $4 \mathrm{~K}$ temperature scattering on ionized impurities dominates at low carrier energy. For electron energies larger than about $100 \mathrm{kT}$ scattering on piezoacoustic phonons and then (higher electron energies) acoustic phonon emission processes dominate. We can only exclude polar optical phonon scattering shown to be unimportant for $4 \mathrm{~K}$ temperature and for expected carrier energies.

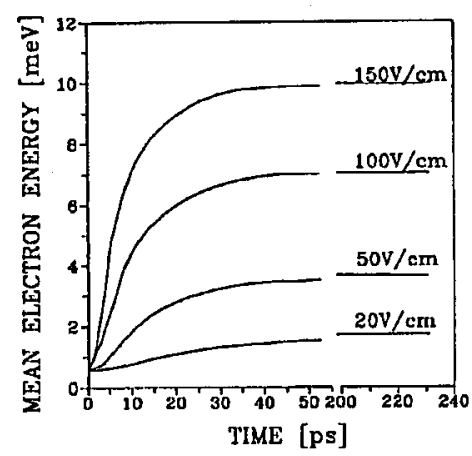

Fig. 1. Carrier heating efficiency for different electric field applied (in $\mathrm{V} / \mathrm{cm}$ units). Electric fields were taken from the range expected in the ODCR experiment. Calculations were performed for $4 \mathrm{~K}$ temperature when optical phonon scattering is not important.

In Fig. 1 we show the calculated carrier heating efficiency for different electric fields expected in the microwave cavity. These calculations were performed for ionized impurities and acoustic phonon (deformation potential and piezoelectric) scattering mechanisms. As mentioned above, scattering on optical phonons is not important for $4 \mathrm{~K}$ temperature and for low electron energies. The results shown in Fig. 1 prove that free carriers can easily accumulate energies sufficient for exciton dissociation and for impact ionization of most of the shallow centers in typical semiconductor. The impact ionization mechanism can thus be responsible for optical detection of the cyclotron resonance, as concluded previously from ODCR studies of silicon $[2,3]$.

One of the most attractive property of ODCR study is the sharpness of thresholds for impact ionization processes. It was observed that ODCR experiment allows one to reach separately the condition for impact ionization of centers of very 

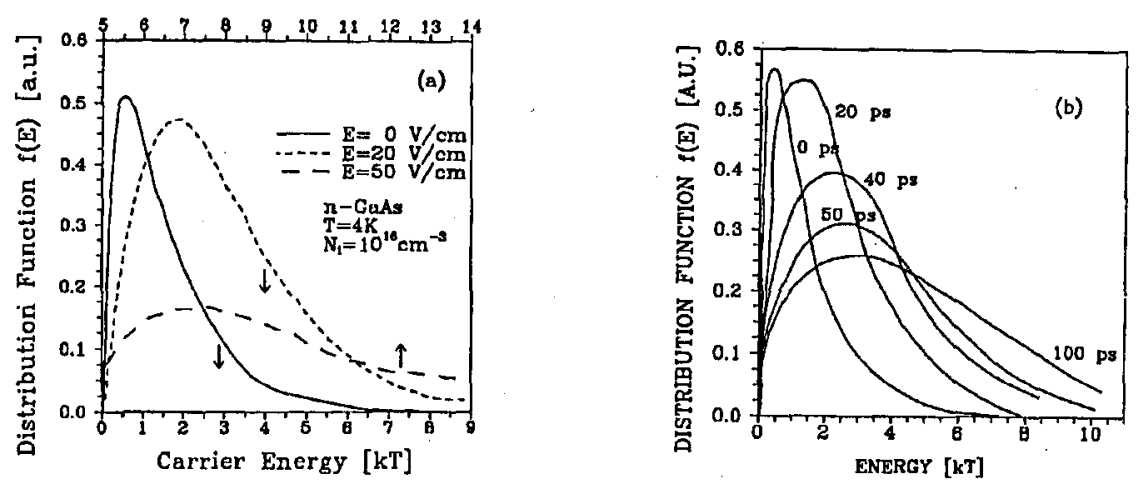

Fig. 2. Energy distribution of CR heated electrons $40 \mathrm{ps}$ after turning on the specified electric field (a). In (b) energy distributions of CR heated electrons for $10 \mathrm{~V} / \mathrm{cm}$ electric field component of microwaves and five different times after turning on the field are shown. Carrier energy is given in normalized units of $\mathrm{kT}$.

similar binding energies. The contribution of two shallow donors to donor-acceptor pair recombination processes could be resolved in that way [6]. Monte Carlo calculations, shown in Fig. 2a,b, explain the physical principles responsible for this property of the ODCR method. It is shown that very sharp distribution of mean electron energy is obtained for carrier heating by low magnitude, high frequency electric field. It is important to notice that not only carrier heating by low electric field is required (Fig. 2a), but high field frequency as well. The latter is explained by the results shown in Fig. $2 \mathrm{~b}$. The energy distribution of mean electron energy smears out in time and broad distribution function is expected for dc impact ionization experiments. For high frequency electric field component of microwaves sharp energy distribution is predicted by our calculations. This is why the ODCR method could find a new application for high spectral resolution photoluminescence experiments and may be applied for high spectral resolution PL stidies of edge emissions in III-V compounds, as reported previously by one of us [6].

\section{References}

[1] R. Romestain, C. Weisbuch, Phys. Rev. Lett. 45, 2067 (1980).

[2] M. Godlewski, H. Weman, F.P. Wang, B. Monemar, W.M. Chen, Q.X. Zhao, in: Defects in Electronic Materials, Eds. M. Stavola, S.J. Pearton, G. Davies, Mater. Res. Symp. Proc., Vol. 104, Material Research Society, Pittsburgh 1988, p. 117.

[3] H. Weman, M. Godlewski, B. Monemar, Phys. Rev. B 38, 12525 (1988).

[4] B.M. Ashkinadze, V.V. Belkov, A.G. Krasinskaya, Sov. Phys. Semicond. 24, 555 (1990).

[5] A. Moll, C. Wetzel, B.K. Meyer, P. Omling, F. Scholz, Phys. Rev. B 45, 1504 (1992).

[6] M. Godlewski, K. Fronc, M. Gajewska, W.M. Chen, B. Monemar, Phys. Rev. B 44, 8357 (1991).

[7] W. Fawcett, A.D. Boardmans, S. Swain, J. Phys. Chem. Solids 31, 1963 (1970).

[8] S. Dedulewicz, K. Karpińska, M. Godlewski, to be published. 